\title{
Editorial
}

\section{The promise of research governance: building research and development capacity and clinical research in primary health care}

For the new primary health care (PHC) organizations in the UK the requirements of research governance may appear to be an additional burden at a time of huge organizational change. In reality the research governance requirements, for example in England (Department of Health, 2001), provide a means both for research and development (R\& D) capacity building to become a mainstream NHS activity and for the expansion of clinically relevant R\&D by this new body of practitioner researchers in PHC.

There has been concern over many years about the lack of R\&D capacity in PHC as well as the need to increase research to underpin PHC practice (Medical Research Council, 1997, NHS Executive, 1997). The introduction of career scientist and other research awards for individuals pursuing a research career in PHC has been enthusiastically welcomed. Such awards and new schemes such as the doctoral and post-doctoral awards for nurses, midwives, health visitors and allied health professionals announced in May, have an important contribution to make to the development of individuals and research teams. Achievement of the full potential of these awards to individuals, however, requires that health care employers provide an environment in which R\&D skills can be utilized to improve practice. Practitioners given the opportunity to develop research skills have been shown to focus on clinical research issues and make significant changes to practice themselves and through impact of their projects on colleagues (Bryar and Bytheway, 1996). In a recent interview Professor Senga Bond, Chair of the Nursing Panel for the 2001 Research Assessment Exercise, criticized nursing academics for their distance from practice and their lack of research concerning clinical topics (News Item, 2002). Career pathways in nursing and other PHC disciplines have restricted the ability of many people interested in research maintaining clinical responsibilities or contact. Outside of medicine, individuals who have sought to maintain a clinical and academic focus may be viewed as mavericks who have pursued opportunistically career changes giving them $R \& D$ and clinical skills.

The research governance framework places a responsibility on NHS employers to develop human resources policies which provide $R \& D$ career paths which are fully integrated with other career paths in the NHS. This requirement will necessitate a review of all job descriptions in primary care organizations to identify the R\&D elements of each post. Questions will have to be asked about how, for example, a post enables an individual to utilize research in their practice, develop R\&D project management skills or utilize R\&D in policy development.

However, changes in job descriptions will not be sufficient. For many years $\mathrm{G}$ grade community nursing job descriptions have made reference to the research utilization and research involvement expected of post holders. What has been lacking has been a culture in primary care organizations which has facilitated or required demonstration of such activities at all levels. Leadership support will be needed to ensure that real R\&D time is available to members of primary care organizations to ensure that $R \& D$ career paths become a reality and that there is a significant increase in research to underpin PHC practice. The new human resource policies will need to have the support of executive teams, managers and team leaders at all levels of the organization. These policies will, of necessity, place $R \& D$ requirements on them as well, to both acquire R\&D skills, make use of research and support clinicians in developing their research practice. 
In addition to changes in human resources policies, primary care organizations will also need to provide opportunities for those developing and using R\&D skills to be part of collaborations and partnerships both within and between organizations. If we examine the research careers of leading researchers in primary care who have maintained their clinical role we find that they were part of research groups and wider networks, with colleagues and often members of their family contributing to the research activity. We tend to think of the individual lone researcher, but the reality is more usually of an individual enmeshed in informative research and clinical networks. Such networks develop an individual's knowledge of $R \& D$ practice; enrich research by bringing new perspectives and provide collaborators who may be able to undertake some R\&D activities when the practitioner researcher has to concentrate on practice issues. This need argues for the maintenance and enhancement of $R \& D$ networks in PHC. It would be a pity, just when $R \& D$ expectations are being built into PHC organizations, if the current $\mathrm{R} \& \mathrm{D}$ networks were weakened. Other changes, such

as the incorporation of public health in primary care organizations, provide the opportunity to extend and enrich R\&D networks both within and between organizations.

At the same time as these changes are taking place in primary care organizations there also needs to be a review within academic departments of their mechanisms both to support practitioners who are developing research skills and academic staff who need to maintain clinical expertize. As Glen (2002) comments, there are a large number of different collaborative relationships between academic departments, the NHS, social services and other organizations which support $R \& D$ capacity building and research. She suggests the need for an audit of such models to identify the most effective in terms of research career pathways and in supporting clinically relevant research.

In conclusion, the research governance framework provides the opportunity for practitioners with R\&D interests to pursue a coherent as opposed to maverick career in PHC combining practice and $R \& D$. It provides the opportunity for the NHS to make full use of the R\&D capacity available within organizations. The development of this PHC R\&D workforce will enable the production of a substantial evidence base for practice. Research governance should therefore be welcomed by all those concerned with $R \& D$ in primary care organizations!

Rosamund Bryar Joint Editor

Primary Health Care Research and Development Professor of Community and Primary Care Nursing

City University, London, UK Email:R.M.Bryar@city.ac.uk

\section{References}

Bryar, R. and Bytheway, B. 1996: Changing Primary Health Care: The Teamcare Valleys Experience. Oxford, Blackwell Science.

Department of Health 2001 Research Governance Framework for Health and Social Care. London, Department of Health.

Glen, S. 2002 Practice/academic career pathways in the health and social care professions. Learning in Health and Social Care, $1(1), 59-60$.

Medical Research Council 1997: Primary Health Care. MRC Topic Review. (Chair Prof. N. Stott), London, Medical Research Council.

NHS Executive 1997: $R \& D$ in primary care. National Working Group report. (Chair Prof. D. Mant). London, Department of Health.

News Item 2002: 'Academics have lost clinical focus' Nursing Times 98(16), 7. 